\title{
THE USA X-RAY TIMING EXPERIMENT
}

\author{
P. S. Ray ${ }^{1}$, K.S. Wood ${ }^{1}$, G. Fritz ${ }^{1}$, P. Hertz ${ }^{1}$, M. Kowalski ${ }^{1}$, W.N. Johnson ${ }^{1}$, \\ M.N. Lovellette ${ }^{1}$, M.T. Wolff ${ }^{1}$, D. Yentis ${ }^{1}$, R. M. Bandyopadhyay ${ }^{2}$, E.D. \\ Bloom $^{3}$, B. Giebels 3 , G.Godfrey ${ }^{3}$, K. Reilly ${ }^{3,4}$, P. Saz Parkinson ${ }^{3,4}$, G. Shabad ${ }^{3,4}$, \\ P. Michelson ${ }^{4}$, M. Roberts ${ }^{4}$, D.A. Leahy ${ }^{5}$, L. Cominsky ${ }^{6}$, J. Scargle ${ }^{7}$, J. \\ Beall $^{8}$, D. Chakrabarty ${ }^{9}$, Y. Kim ${ }^{10}$
}

1) E. O. Hulburt Center for Space Research, Naval Research Laboratory, 2) NRC Research Associate, 3) Stanford Linear Accelerator Center, 4) Stanford University, 5) University of Calgary, 6) Sonoma State University, 7) NASA Ames, 8) Saint John's College, 9) MIT, 10) Saddleback College

\begin{abstract}
The USA Experiment is a new X-ray timing experiment with large collecting area and microsecond time resolution capable of conducting a broad program of studies of galactic X-ray binaries. USA is one of nine experiments aboard the Advanced Research and Global Observation Satellite which was launched February 23, 1999. USA is a collimated proportional counter X-ray telescope with about $1000 \mathrm{~cm}^{2}$ of effective area per detector with two detectors sensitive to photons in the energy range 1-15 keV. A unique feature of USA is that photon events are time tagged by reference to an onboard GPS receiver allowing precise absolute time and location determination. We will present an overview of the USA instrument, capabilities, and scientific observing plan as well as the current status of the instrument.
\end{abstract}

\section{INTRODUCTION}

The Unconventional Stellar Aspect (USA) Experiment is a low-cost X-ray timing experiment with the dual purpose of timing X-ray binary systems and exploration of applications of X-ray sensor technology. USA was launched on February 23, 1999 on the Advanced Research and Global Observation Satellite (ARGOS). It is a reflight of two proportional counter X-ray detectors that performed excellently on the NASA Spartan-1 mission (Kowalski et al. 1993). The primary targets are bright Galactic Xray binaries that are used simultaneously for both scientific and applied objectives. X-ray photon event times are measured to high precision using the GPS receiver on ARGOS. USA has the effective area, precise timing ability, and data throughput capability to probe these sources at the timescales of processes near neutron star surfaces or the innermost stable orbits around black holes. A second objective of the experiment is to conduct experiments involving applied uses of X-ray detectors in space and with reliable computing in space. These will not be discussed here but descriptions are available elsewhere (Wood 1993).

Key characteristics of the experiment and mission that facilitate this overall 
program include (i) a mission concept that allows long observing times on bright Xray objects, (ii) large-area detectors with high time resolution capability (effective area: $2000 \mathrm{~cm}^{2}$; telemetry: $40 \mathrm{kbps}$, with $128 \mathrm{kbps}$ available for short periods; $2 \mu \mathrm{s}$ time resolution), (iii) good low energy response (down to $1 \mathrm{keV}$ ), and (iv) a high flexibility in data handling. Other special features include absolute time-tagging (to $2 \mu \mathrm{s}$ ) using a GPS receiver.

\section{SCIENTIFIC PROGRAM}

The principal targets for USA are X-ray binaries whose X-ray emitting members are neutron stars, black holes, or white dwarfs. Study of physical processes in these systems have been among the main thrusts of X-ray astronomy since the founding of the field. Today it remains true that many of the most important results on these systems are found by studying their X-ray variability, and the push to shorter (millisecond) timescales is proving highly fruitful. If the source is bright ( $>$ milliCrabs) such short timescales are more readily reached with non-imaging instruments having large collecting apertures than with imaging instruments. Physics issues studied in these sources are generally related to the fact that parameters such as magnetic field strength, mass and energy densities, and gravitational fields reach extreme values, hence providing the preferred testing grounds for physical theories. X-ray timing is a cornerstone of relativistic astrophysics.

USA, in turn, is one of the two main resources at the present epoch for X-ray timing experiments, the other being the Proportional Counter Array (PCA) on RXTE. USA has its own special areas of emphasis, one of which is its observing plan. Present plans call for the observation of about 30 primary targets, with each being observed for about 1 month over a nominal mission life of 3 years; selected targets will be observed for shorter periods of time. Sources observed to date (through 31 August 1999) include Cyg X-1 (700 ks on target), Aql X-1 (100 ks), Cen X-3 (65 ks), X1630-472 (60 ks), Cyg X-2 (50 ks), X1636-536 (45 ks), GX 1+4 (40 ks), 1E2259+586 (40 ks), X1820-30 (40 ks), X1630-472 (35 ks), 1E1048.1-5937 (30 ks), and GRS $1915+105(25 \mathrm{ks})$. The total time on each source is typically scheduled as a number of $\sim 1$ ks observations distributed over weeks or months. Simultaneous observation with other observatories, such as the Compton Gamma Ray Observatory and Rossi X-ray Timing Explorer, and with ground based telescopes are also being undertaken.

Figure 1 shows two sample light curves taken with USA. The first is an X-ray burst from the burster X1735-444, and the second is an observation of a flaring state of the Galactic microquasar GRS1915+105. In 1735-44 the instrument is on the source throughout the interval displayed while in GRS 1915+105 the steep rise at the beginning of the plot is the instrument slewing onto the source; the earliest seconds represent the background for this observation. 
$\times 1735-444$

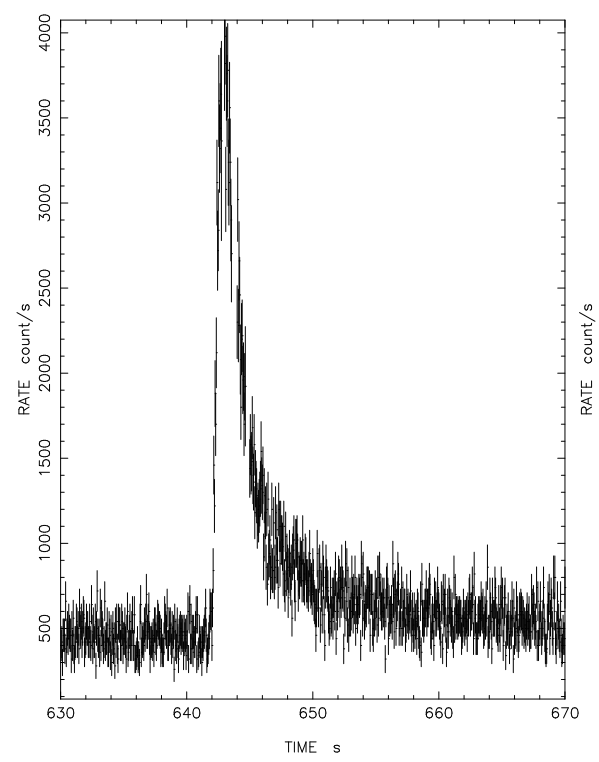

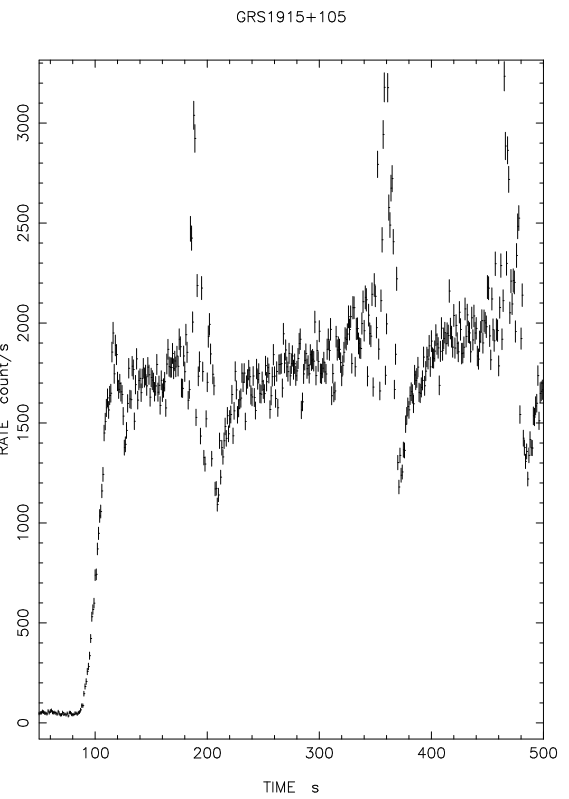

FIGURE 1. Left: A burst from X1735-444. Right: An observation of GRS1915+105

\subsection{Low-Mass X-ray Binaries}

The special importance of the low mass X-ray binaries (LMXBs) arises from their comparatively weak magnetic fields which allows the disk to penetrate very close to the star. This gives rise to fast timing effects that can be used to probe the extreme conditions in the neutron star vicinity. Major gains in the understanding of these phenomena have been made since the launch of the Rossi X-ray Timing Explorer (RXTE) in late 1995. High frequency quasiperiodic oscillations (QPOs) and short strings of coherent pulsations during bursts have been used to argue convincingly that effects associated with inner disk edges and the innermost stable orbits predicted by General Relativity are being seen (van der Klis 1998). Another milestone is the establishment of the spinup evolution of neutron stars through the discovery of the first accretion powered millisecond pulsar (SAX J1808.4-3658).

USA will make further contributions to the study of LMXBs with the application of its unique strengths. In some cases this will mean exploiting the ability to dedicate large blocks of time to a key source, e.g., to refine understanding of SAX J1808.4-3658 or to observe transitions between modes or states. Significant time is being devoted to searches for coherent periods, both on and off bursts. Off burst work is carried out using coherence recovery searches for periods. Observations can also be carried out in various ways to detect or refine orbital periods in LMXBs. 
Overall, LMXBs are sources that stand to bring major rewards including advances in understanding the role of General Relativity in the dynamics of inner disk regions, but past experience has also shown that these rewards are achieved only through major investments of observing time and analysis, chiefly because of the elusiveness and short timescale of the spin periods.

\subsection{High-Mass X-ray Binaries}

USA will also accumulate significant time on a number of high-mass X-ray binaries. Many of these systems have accretion rates that far exceed the Eddington limit locally in the accretion column. This means that radiation pressure has a significant influence on the flow. Recently Jernigan et al. (1999) reported the discovery of Photon Bubble Oscillations (PBOs) in Cen X-3. USA will observe this source and other bright HMXBs to help characterize their high frequency power spectra independently from RXTE. Outstanding puzzles in these systems are the details of angular momentum transfer from the disk to the star (including possible reversals of the sense of disk rotation) and understanding in detail the photohydrodymics of the accretion column in which the super Eddington accretion funneled flow is converted to the observed X-ray emission. Bright binary pulsars such as Her X-1, Cen X-3, and Vel X-1 will be observed to gain insight into these issues. Monitoring over both short and long time periods allows the correlation between period, period derivative, and luminosity to be probed which addresses the angular momentum transfer issue. USA observes at significantly lower energies than the BATSE instrument on CGRO, which has gathered much of the data on this topic in recent years.

\subsection{Black Hole Candidates}

USA will pursue several investigations into the nature of black holes. Chief among these are (1) The characterization of high frequency $(\nu>1 \mathrm{kHz})$ variability in Cyg $\mathrm{X}-1$. USA has begun a long term study of Cyg X-1 in which more than 1 Ms of exposure will be accumulated on Cyg X-1 in each of the spectral states it exibits during the USA three year mission. Using techniques developed for calibrating high frequency systematic effects in HEAO A-1 and RXTE (Chaput et al. 1999), we will constrain the sub-millisecond variability of Cyg X-1. (2) Simultaneous X-ray and infrared observations of the galactic microquasar GRS 1915+105. USA will, for the

first time, determine the 1-3 keV behavior of this interesting source. Figure 1 does not begin to convey the range of modulation patterns seen in this object. A new window at lower energies from USA, gathered in simultaneity with other space-based and ground facilities, may contribute to modeling the fluid dynamical processes near the black hole. The model that can account for the wealth of variability effects seen in GRS 1915+105 may bring us closer to understanding how plasma behaves near black holes, including the relativistic effects on orbits. 


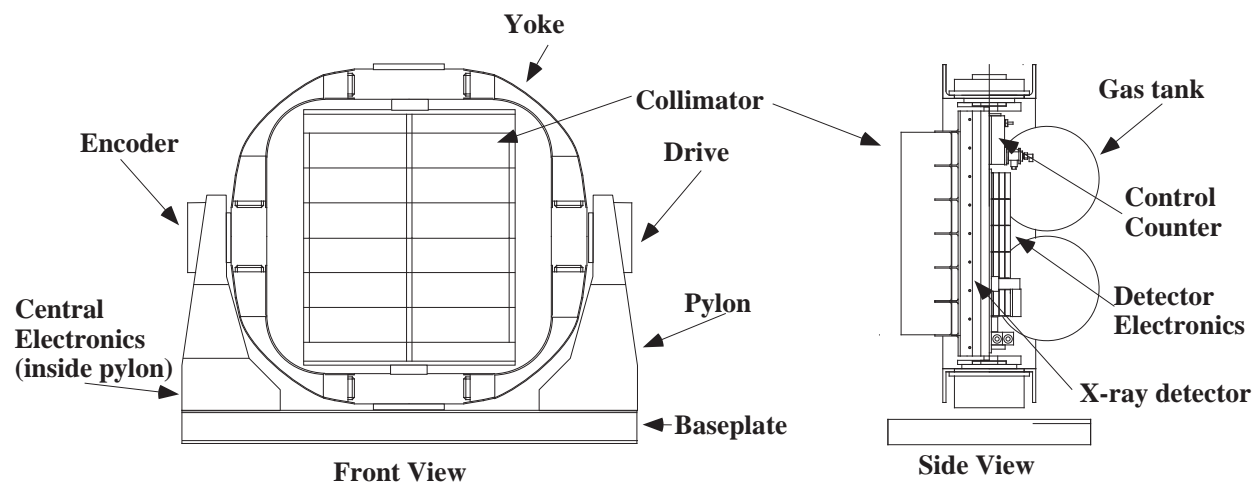

FIGURE 2. Two Views of the USA Experiment

\subsection{Other Sources}

The Anomalous X-ray Pulsars (AXPs) appear to be a distinct subpopulation whose observed emission is powered by spin-down. Timing these pulsars over a period of years will place strong constraints on their nature (whether they are magnetars or accreting neutron stars) and emission mechanism. Subtle effects of (currently unsuspected) binary companions may show up or else derivatives that provide insight into source dynamics may be measured. There are several good candidates and the result will not necessarily be the same in all instances. Long-term monitoring of AXPs is made feasible by the soft response of USA and the good absolute timing. Rotation-powered (radio) pulsars are also observed by USA to validate the time transfer between USA and RXTE, and to measure the radio to X-ray offset of the pulses.

Cataclysmic variables (CVs) exhibit a wide range of timing phenomena,including QPOs, X-ray transients, and complex light curves. While CVs are typically $\sim 100$ times fainter than LMXBs, their dynamical time scales are $\sim 1000$ times longer. Moreover the magnetic CVs, which will be USA's prime targets among CVs are distinguished by having the largest magnetic moments among known stellar populations, including even magnetars. Curiously, accretion-induced QPOs were predicted historically to result from this flow before they were observed. The QPOs have been seen repeatedly in optical wavelengths but never in X-rays, despite searches. Highly correlated optical and X-ray luminosity variations are predicted in current hydrodynamic models (Wolff, Wood \& Imamura 1991)

Finally, USA can exploit its great flexibility to observe targets of opportunity that are deemed important by the science working group. Already, USA has observed Aql X-1, the Rapid Burster, and X1630-472 during outbursts. Of course, the accreting millisecond pulsar SAXJ1808.4-3658 would be of particular interest if it becomes active during the USA mission. 
TABLE 1. Operational Features of the USA Detector System

\begin{aligned} & \hline Gas: P-10 at 1.1 atmosphere \\ & Flow rate: $0.1 \mathrm{cc} /$ min allows for $3-6$ year life \\ & Window: $5.0 \mu \mathrm{m}$ Mylar $+\sim 30-40$ ANichrome \\ & Energy range: $1-15 \mathrm{keV} \\ &$ Field of view: collimation of $1.2^{\circ} \times 1.2^{\circ}(\mathrm{FWHM}) \\ &$ Energy resolution: $0.17(1 \mathrm{keV} @ 5.9 \mathrm{keV}), 128 \mathrm{raw}$ PHA channels \\ & Aperture (effective): $2000 \mathrm{~cm}^{2} @ 3 \mathrm{keV} \\ &$ On-orbit calibration: solenoid operated ${ }^{55} \mathrm{Fe}$ source \\ & \hline\end{aligned}

\section{INSTRUMENT DESCRIPTION}

\subsection{Proportional Counter X-ray Detectors}

The detector (Table 1) consists of two multiwire constant flow proportional counters equipped with a $5.0 \mu \mathrm{m}$ Mylar window and an additional $1.9 \mu \mathrm{m}$ thick aluminized Mylar heat shield. The detector is filled with a mixture of $90 \%$ argon and $10 \%$ methane (P-10) at a pressure of 16.1 psia (at room temperature). The detector interior contains an array of wires which provides two layers of nine $2.8 \mathrm{~cm}$ square cells, each containing one anode wire, running the length of the counter. An additional wire runs around the periphery of the array as part of the cosmic ray veto system. The electronics are designed to accept primarily X-ray events, which arise in one cell only. Events registered in two or more wires by a cosmic ray track are vetoed with an efficiency of about $99 \%$.

The high voltage on the anode wires is adjusted continuously to stabilize the gain, using a feed-back loop which monitors the pulse-height distribution of $\mathrm{X}$ ray events in a small separate proportional counter. Two discriminators provide a normalized value independent of the absolute source intensity of the ${ }^{55} \mathrm{Fe}$ source used in the feed-back counter.

The collimators serve to support the window as well as to define the field of view. To place reasonable requirements on the pointing system the collimator was constructed with a field of view of approximately $1.2^{\circ} \times 1.2^{\circ}$ and a flat top of approximately $0.05^{\circ}$. Each collimator consists of 8 modules $7.5 \mathrm{~cm} \times 28 \mathrm{~cm} \times 11 \mathrm{~cm}$ high filled with copper hexcell formed from $25 \mu \mathrm{m}$ sheet stock with a $2.5 \mathrm{~mm}$ altitude for each hexagon. The sides and ends of each module are formed from $1 \mathrm{~mm} \mathrm{Cu}$ sheet which provides stiffness across the width of the collimator to support a $98 \%$ transmission nickel mesh that in turn supports the Mylar window. The response function of each collimator module was measured with X-rays before the modules were assembled into the collimator frames. 
TABLE 2. Pointing System Characteristics

\begin{aligned} & \hline Pitch/Yaw drive capability: $\sim 3.6^{\circ} / \mathrm{min}$ (track), $\sim 20^{\circ} / \mathrm{min}$ (slew) \\ & Field of Regard: $2 \pi \mathrm{Sr} \\ &$ Common pitch/yaw pivot design: $180^{\circ}$ travel in each axis \\ & Drive system: $1.8 \mathrm{deg}$ stepper motor \\ & $800: 1$ gear reduction, $\sim 20^{\prime \prime} / \mathrm{step} \\ &$ Position sensing system: 16 -bit optical shaft encoder \\ & \hline\end{aligned}

\subsection{Support Hardware}

The ARGOS spacecraft is three-axis-stabilized and nadir-pointed. The X-ray detectors are mounted on a 2-axis gimballed platform to permit inertial pointing at celestial objects. The pointer is configured as an equatorial mount looking aft (away from the velocity vector of the spacecraft). Pointing is accomplished by a yaw rotation to acquire the target followed by a continuous slew in the pitch to track the target as it rotates about the orbital pole.

The primary tasks of the central command and control electronics (CE) are command and data interface to the ARGOS spacecraft MIL-1553 bus, data acquisition from the detector modules, control of the pointer system, and interface with USA's RH3000 and IDT3081 processors. The command and control processor is a Harris radiation hardened $80 \mathrm{C} 86$ microprocessor.

The structural elements of the pointer (Table 2), the support pylons, and the yoke which serves as the inner gimbal form the primary structure of the experiment. Each axis has a drive unit which forms the pivot on one end of the axis and a position encoder unit which supports the opposite end. Actual alignment is measured by rastering through sources in flight.

The detector interface board (DIB) in the CE performs time tagging and data formatting for the X-ray science data as well as formatting detector housekeeping data. The microprocessor used is an Analog Devices ADSP2100. The DIB receives a fast photon arrival signal from each detector which enables the timing to $\sim 1$ microsecond accuracy. A $1 \mathrm{~Hz}$ clock (with corresponding GPS time tag) is received directly from the spacecraft to synchronize the event time tagging clock. Pulse height data for each photon are transmitted from the detector to the DIB upon completion of the analog to digital conversion. There are two standard telemetry modes: event and spectral. Event mode is the "workhorse" telemetry mode for USA; for moderate count rates, it allows the maximal amount of information to be preserved on each photon. In event mode, the arrival time and some energy information is stored for each photon detected. There are two submodes of event mode providing $32 \mu$ s time and 16 pulse height channels in a 12 bit word and $2 \mu$ s time with 8 pulse height channels in a 15 bit word respectively. Data may be output in event mode at either 40 or 128 kbps providing maximum count rates of 3060 or 9940 events per second 
for $32 \mu$ s time or 2448 or 7952 events per second for $\mu$ s time. In spectral mode, a full resolution energy spectrum (48 channels) is generated every 10 milliseconds for each detector.

The USA experiment also provides space for two "ride-along" processor boards, the RH3000 and the IDT3081. The RH3000 board is built around a pair radiation hardened Harris Semiconductor version of the MIPS R3000 configured as a shadow pair with $2 \mathrm{MB}$ of memory. The IDT3081 board incorporates the commercial-off-theshelf IDT3081 processor and $2 \mathrm{MB}$ of DRAM without any special error correcting hardware. Both computer boards have access to the downlink science telemetry stream. These processors will be used to conduct experiments in fault-tolerant computing, autonomous spacecraft navigation, and to perform special data analysis functions which are beyond the scope of the normal science telemetry modes, or which require bandwidths greater than $128 \mathrm{kbps}$.

\subsection{Instrument Status}

The USA instrument has been performing well since activation began on 30 April 1999, but the USA mission has not been without its difficulties. Approximately two weeks after launch the detector heat shields suffered from degradation which has imposed additional constraints on USA pointing with respect to the Sun. On 8 June 1999 Detector 2 suffered an event which increased the gas leak rate to a very high level and exhausted the P-10 supply leaving only Detector 1 to complete the mission, halving the effective area. Two spacecraft performance issues which are described in more detail below have also impacted USA operations.

\section{ARGOS MISSION DESCRIPTION}

USA exploits the flight opportunity provided by the ARGOS mission under the DoD Space Test Program (STP). STP was established in 1965 as an activity under the executive management of the Air Force Systems Command with the objective of providing spaceflight for DoD research and development experiments which are not authorized to fund their own flight. Both engineering/technology development and scientific payloads have been flown with great frequency under this program. ARGOS is the only Delta-class STP free-flyer mission to be launched in the 1990s.

The $5000 \mathrm{lb}$ ARGOS satellite was launched from Vandenberg AFB at 10:30 UT on 23 February 1999 aboard a Boeing Delta-II rocket. The prime satellite contractor was Boeing who built and tested the satellite at their Seal Beach, CA facility. ARGOS carries a complement of 9 experiments which address such topics as ionospheric remote sensing, space dust, advanced electric propulsion, and high temperature superconductivity.

Spacecraft downlink telemetry bandwidth is done at 1, 4, or 5 Mbps. Data are stored in a 2.4 Gbit solid state recorder and downlinked at station passes to AFSCN ground stations. The spacecraft is operated in a 3 -axis stabilized mode, with Z-axis of the spacecraft always pointed to nadir. Attitude control is based on a 
system of gyros and horizon sensors feeding into reaction wheels and $\mathrm{CO}_{2}$ thrusters. The orbit is nearly circular with a $830 \mathrm{~km}$ altitude and a $98.7^{\circ}$ inclination. It is Sun-synchronous with a beta-angle of 25-45 degrees, i.e., it crosses the equator at approximate local times of 14:00 on the day side and 02:00 on the night side. This nearly polar orbit means that USA encounters a high radiation environment multiple times per orbit as it passes through the Earth's radiation belts at latitudes above $50^{\circ}$. This forces USA to take data at lower duty cycle, turning off the detectors in the radiation belts and the South Atlantic Anomaly to prevent detector breakdowns.

\subsection{Mission Operations and Data Processing}

The satellite mission operations are handled by the Air Force SMC/TEO at Kirtland AFB, NM. They are responsible for uplinking commands to and for receiving data from the satellite. Individual experiment command uploads are delivered to TEO via FTP and uploaded during ground contacts. Data downlinked during the pass is recorded at the ground station and mailed to TEO because the AFSCN does not support real time links of $>1 \mathrm{Mbps}$. This results in a delay of $7-21$ days in getting science data back to the experimenters.

USA operation is largely automatic. Twice daily command uploads contain timed execution commands to slew the insrument, command it to track the source, switch on the high voltage, select telemetry rate and perform calibrations. These command sequences are generated by a highly automated observation scheduling system which optimizes source selection, manages solid state recorder space, and builds the command uploads.

The USA data processing system is also highly automated. As data appear at Kirtland, files are automatically retreived via FTP and the first several processing steps are performed. Quicklook data are checked for anomalous conditions and the USA team is alerted by e-mail if problems occur. Subsequently, observations are extracted from the Level 0 archive, converted to FITS, and distributed to the scientific analysis centers, including NRL and SLAC.

A Science Working Group (SWG) has been established to help optimize the scientific potential of USA. The SWG determines scientific priorities for observing targets, subject to certain constraints. Scheduling of targets during the USA mission will be consistent with experiment science objectives, priorities, and mission operations capabilities. Telemetry formats will be selected to support overall objectives. USA has the flexibility to respond quickly to some targets of opportunity with approximately a 1-3 day turnaround after the decision to revise the observing plan. The SWG decides whether to respond to potential targets of opportunity and also identifies instances when coordinated observations with ground-based observers, CGRO, RXTE, or other ARGOS instruments are scientifically advantageous. The USA team is receptive to collaborations to make better use of the data, but the small size of the group does not allow us to operate a conventional guest observer facility. 


\subsection{ARGOS Mission Anomalies and Events}

The ARGOS launch and deployment went flawlessly, but since then several problems have surfaced with various subsystems. Generally, the spacecraft has been very robust and has autonomously safed itself when presented with dramatic disturbances, such as a battery exploding on the electric propulsion experiment and during the USA Detector 2 gas leak. Here we will just summarize the issues and describe how they affect the operation of USA.

Shortly after launch, it was discovered that the GPS receiver is unable to stay locked on to the GPS solution and provide good navigation information. This problem was traced to an unexpectedly large input level to the receiver which causes cross correlation errors which disrupt the solution. Generally the receiver will lock on, then oscillate between navigation and acquisition mode for a period of a few minutes to a few hours before losing the solution completely. To recover the time resolution required for many of the USA objectives, new software was uploaded to the satellite to make it safer to initialize the receiver repeatedly. Currently the receiver is initialized 4 times per day. Software is being developed to be able to interpolate times using the onboard clock to recover precise absolute times between periods when the receiver is locked on to a good solution.

A problem was discovered with the Scanning Horizon Sensors which are used to control the pitch and roll of the satellite. They are more radiation sensitive than expected and experience data dropouts or return incorrect data during most passages through the South Atlantic Anomaly (SAA). This causes the spacecraft to respond and produces attitude disturbences in the satellite when it is in the SAA. This does not affect USA because USA never operates in the SAA.

At the time of the software upload to work around the GPS receiver problem, a problem with the offset pointing of USA from the satellite appeared. It appears that the navigation message sent to USA every second no longer represents the true attitude of the spacecraft. It appears, after numerous scanning observations using USA, that the satellite is out of alignment in the roll direction by about $1^{\circ}$. The cause of this is currently unknown, and work is ongoing to troubleshoot this problem and design a workaround.

\section{REFERENCES}

Chaput, C., Bloom, E., Cominsky, L., et al. 1999, ApJ, submitted (astro-ph/9901131)

Jernigan, J. G., Klein, R. I., Arons, J. 1999, ApJ, in press astro-ph/9909133)

Kowalski et al. 1993, ApJ, 412, 489

van der Klis, M. 1998, in Proceedings of the Third William Fairbank Meeting

Wolff, M. T., Wood, K. S., \& Imamura, J. N. 1991, ApJ, 375, L31

Wood, K. S., et al. 1991, ApJ, 379, 295

Wood, K.S. 1993, SPIE 1940, 105 